Original Article

\title{
Effect of home-based training using a slant board with dorsiflexed ankles on walking function in post-stroke hemiparetic patients
}

\author{
Yasuhide Nakayama, RPT, MS, PhD ${ }^{1)^{*}}$, Setsu IiJima, MD, $\mathrm{PhD}^{2)}$, \\ Wataru KaKuda, MD, $\mathrm{PhD}^{1)}$, Masahiro Abo, MD, $\mathrm{PhD}^{1)}$ \\ 1) Department of Rehabilitation Medicine, Jikei University School of Medicine: 3-25-8 Nishi-Shimbashi, \\ Minato-Ku, Tokyo 105-8461, Japan \\ 2) Rehabilitation Services Bureau, National Rehabilitation Center for Persons with Disability, Japan
}

\begin{abstract}
Purpose] To investigate the effects of a 30-day rehabilitation program using a slant board on walking function in post-stroke hemiparetic patients. [Subjects and Methods] Six hemiparetic patients with gait disturbance were studied. The patients were instructed to perform a home-based rehabilitation program using a slant board, thrice daily for 30 days, the exercise included standing on the slant board for 3 minutes, with both ankles dorsiflexed without backrest. For all patients, the Brunnstrom Recovery Stage, Barthel Index, range of motion of the ankle joint, modified Ashworth scale scole for calf muscle, sensory impairments with Numeral Rating Scale, maximum walking speed, number of steps, and Timed "Up and Go" test were serially evaluated at the beginning and end of the 30-day program. [Results] The program significantly increased walking velocity, decreased the number of steps in the 10-m walking test, and decreased Timed "Up and Go" test performance time. [Conclusion] This rehabilitation program using the slant board was safe and improved walking function in patients. The improvement in walking function could be due to a forward shift of the center of gravity, which can be an important part of motor learning for gait improvement.

Key words: Stroke, Inclined surface, Walking function
\end{abstract}

(This article was submitted Mar. 11, 2016, and was accepted May 14, 2016)

\section{INTRODUCTION}

Stroke is a leading cause of long-term disability, and the absolute number of patients with stroke is increasing. Of the neurological sequelae that cause functional disability, hemiparesis is the most common. The incidence of gait disturbance due to hemiparesis is reportedly relatively high among chronic stroke patients ${ }^{1,2}$. The occurrence of this disability leads to marked impairment of quality of life and the sense of well-being ${ }^{3,4}$. In addition, the burden of caregivers is anticipated to increase when patients are in need of assistance for walking ${ }^{5}$. Improved walking ability is one of the most common goals for post-stroke hemiparetic patients ${ }^{6,7)}$. A slant board has been used as a therapeutic device for patients with spastic lower hemiparesis. Standing on the toe-up inclination surface stretches the calf muscles, which reduces the pathologically increased lower limb muscle tone in post-stroke hemiparetic patients. Standing without a back rest on the slant board can produce a forward shift in the center of pressure in healthy adults and hemiparetic patients, as reported in our previous study. Kluzak reported that standing on an inclined surface resulted in an after-effect of learning in healthy, blindfolded subjects when they returned to standing on a horizontal surface ${ }^{8}$. Subjects leaned forward after they stood on a toes-up inclination surface. Recent reports described other benefits of using the board in healthy adults and hemiparetic patients, such as movement of "center of pressure" "), and possible increase in the maximum range of "center of gravity" in the antero-posterior direction"

*Corresponding author. Yasuhide Nakayama (E-mail: pt_nakayama@jikei.ac.jp)

(C)2016 The Society of Physical Therapy Science. Published by IPEC Inc.

This is an open-access article distributed under the terms of the Creative Commons Attribution Non-Commercial No Derivatives (by-nc-nd) License $<$ http://creativecommons.org/licenses/by-nc-nd/4.0/>. 
Neurophysiological studies have also demonstrated increased contraction of the anterior tibialis muscle in healthy subjects standing on the slant board ${ }^{11)}$. Daily home-based rehabilitation using the slant board was hypothesized to improve gait in post-stroke hemiparetic patients. To test the hypothesis, the present study investigated the effects of a 30-day home-based daily rehabilitation program using the slant board on walking function in post-stroke hemiparetic patients.

\section{SUBJECTS AND METHODS}

The study subjects were 6 post-stroke hemiparetic patients with gait disturbances. The intervention and study protocol were approved by the ethics committee of Jikei University School of Medicine (24-274 7040), and informed consent was obtained from all patients before beginning the study. The following were the inclusion criteria: 1) clinical confirmation of gait disturbance (unable to walk as before the onset of stroke) and Brunnstrom recovery stage for lower limb classification of $3-5 ; 2$ ) ability to walk without the use of canes or orthoses; 3) more than six month after stroke onset; 4) history of a single symptomatic stroke only (no bilateral cerebrovascular lesions). 5) Independency on daily living and no physical or mental illness requiring medical management; 6) no previous local treatment for lower limb spasticity with motor point block or botulinum toxin injections. Table 1 summarizes the clinical characteristics of the study patients. The time between stroke onset and the intervention ranged from 9 to 18 months. Stroke was classified into cerebral infarction in 2 patients and intracerebral hemorrhage in 4 patients.

After the initial evaluation of walking function, the patient was instructed to perform the home-based training using a slant board. Follow-up evaluation was performed 30 days later after the initial evaluation to assess the effect of the training program on walking function. The slant board "Asahi Stretching Board" (ASAHI Corporation, Tokyo, Japan) has a plastic construction and the angle of the slant was set at 20 degrees for all patients (Fig. 1). The home-based rehabilitation program using the slant board was explained in detail to the patients at the beginning of the program. The patient was instructed to step up on the board. The standing position was maintained with both ankle joints dorsiflexed for 3 minutes. During each session, the patient was instructed to perform two sets of 3-min training using the slant board with $>3$ min of rest between the tasks, three times a day (in the morning, daytime, evening) and to perform this protocol daily for 30 days. No other physical training for gait disturbance was recommended. For safety monitoring, the patients were required to immediately inform the hospital by telephone in case of adverse events. At the end of the 30-day protocol, self-reported questionnaires were used to evaluate whether the patients performed and completed the daily protocol as instructed. For all patients, the Brunnstrom Recovery Stage (BRS), Barthel Index (BI), range of motion (ROM) in the ankle joint, modified Ashworth scale (mAs) of calf muscle, sensory impairments with Numeral Rating Scale (NRS), maximum walking Speed (MWS), number of steps and Timed "Up and Go" test (TUG) were serially evaluated at the beginning and end of the 30-day protocol. Walking velocity is a reliable objective measure of walking function and has been reported to correlate with more sophisticated methods used to assess walking function ${ }^{12}$. It was measured and recorded over a $10-\mathrm{m}$ walkway. During measurement, the patient was not allowed to use walking canes. In addition, any assistance was not permitted during the evaluation. First, patients walked $10 \mathrm{~m}$ at a self-selected comfortable pace. Second, they were instructed to walk on the same walkway as fast and as safely as possible. The walking velocity in these trials was recorded as "comfortable walking velocity" and "maximum walking velocity", respectively. The number of steps during the 10-m walking was also counted simultaneously. The TUG is a simple and rapid functional mobility test ${ }^{13}$. Using a series of motor tasks, the test evaluates balance control, muscle strength, and coordination. The reported reliability of the test is excellent, with high intra-class correlation coefficients. In this test, patients are instructed to stand up from a chair with armrests, walk 3 meters, turn around, return to the chair, and sit down. The time taken to complete this task represented the TUG performance time. The time was measured when turning towards the paralytic side as well as towards the non-paralytic side. For all evaluations, two consecutive measurements were taken at each time point and the average value was calculated and recorded.

Data are expressed as mean $\pm \mathrm{SD}$. Differences in the applied measures before and after the 30-day protocol were examined

Table 1. Clinical characteristics of the studied patients

\begin{tabular}{cccccccccccc}
\hline $\begin{array}{c}\text { Patient } \\
\text { No. } \begin{array}{c}\text { Age at } \\
\text { intervention } \\
(\mathrm{yrs})\end{array}\end{array}$ & Gender & $\begin{array}{c}\text { Time after } \\
\text { stroke (m) }\end{array}$ & $\begin{array}{c}\text { Stroke } \\
\text { subtype }\end{array}$ & Handedness & $\begin{array}{c}\text { Side of } \\
\text { hemiparesis }\end{array}$ & $\begin{array}{c}\text { BRS for } \\
\text { lower } \\
\text { limbs }\end{array}$ & $\begin{array}{c}\text { Barthel } \\
\text { index }\end{array}$ & $\begin{array}{c}\text { ROM of } \\
\text { the Ankle } \\
\text { Joint }\left({ }^{\circ}\right)\end{array}$ & $\begin{array}{c}\text { MAS for } \\
\text { plantar } \\
\text { flexors }\end{array}$ & $\begin{array}{c}\text { NRS for } \\
\text { lower } \\
\text { limbs }\end{array}$ \\
\hline 1 & 50 & Male & 9 & CI & Right & Right & 3 & 100 & 30 & 2 & 7 \\
2 & 52 & Male & 10 & CI & Right & Right & 3 & 100 & 30 & 2 & 8 \\
3 & 66 & Male & 12 & ICH & Right & Right & 5 & 100 & 35 & $1+$ & 7 \\
4 & 65 & Male & 18 & ICH & Left & Right & 4 & 100 & 35 & $1+$ & 5 \\
5 & 59 & Male & 16 & ICH & Right & Right & 5 & 100 & 25 & $1+$ & 10 \\
6 & 60 & Male & 11 & ICH & Right & Right & 4 & 100 & 25 & $1+$ & 8 \\
\hline
\end{tabular}

CI: cerebral infarction, ICH: intracerebral hemorrhage, BRS: Brunnstrom recovery stage, BI: Barthel Index, MAS: modified Ashworth scale, NRS: Numeral Rating Scale, ROM: Range of Motion 
for statistical significance using the signed Wilcoxon rank sum test for paired samples. All statistical analyses were performed using SPSS package 17.0 (SPSS, Chicago, IL, USA). A p value less than 0.05 was considered statistically significant.

\section{RESULTS}

At least $89 \%$ of the scheduled training time was completed by all patients over the 30 days. None of the patients experienced pathological symptoms or any deterioration of walking function during the study period. All patients reported that homebased training was not particularly stressful and the training did not interfere with their activities of daily living. Comfortable walking velocity increased significantly from $0.72 \pm 0.14 \mathrm{~m} /$ at baseline to $0.83 \pm 0.26 \mathrm{~m} /$ after the 30 -day program $(\mathrm{p}<0.05)$. Similarly, the maximum walking velocity increased significantly from $0.98 \pm 0.32 \mathrm{~m} /$ at baseline to $1.25 \pm 0.31 \mathrm{~m} /$ after the training $(\mathrm{p}<0.05$, Table 2$)$. The number of steps increased significantly when measured at comfortable walking velocity (baseline: $22.3 \pm 3.3$ steps, end of program: $20.4 \pm 3.6$ steps, $p<0.05$ ) as well as at maximum walking velocity (baseline: 19.5 \pm 4.2 steps, end of program: $17.1 \pm 3.3$ steps, $\mathrm{p}<0.05$, Table 2 ). Furthermore, TUG performance time decreased significantly in tests involving turning towards the paralytic side (baseline: $14.0 \pm 5.3$ seconds, end of program: $10.2 \pm 2.3$, $\mathrm{p}<0.05$ ). In addition, the TUG also decreased significantly in tests involving turning towards the non-paralytic side (baseline: $14.0 \pm 5.6$ seconds, end of program: $10.1 \pm 2.2, \mathrm{p}<0.05$, Table 2 ). Other evaluation results were not significantly different.

\section{DISCUSSION}

This study showed that home-based training using a slant board is safe and significantly improves walking function in post-stroke hemiparetic patients. To our knowledge, this is the first study that reports the beneficial effects of home-based slant board-rehabilitation program for gait improvement for post-stroke patients. In this study, all patients showed improvement

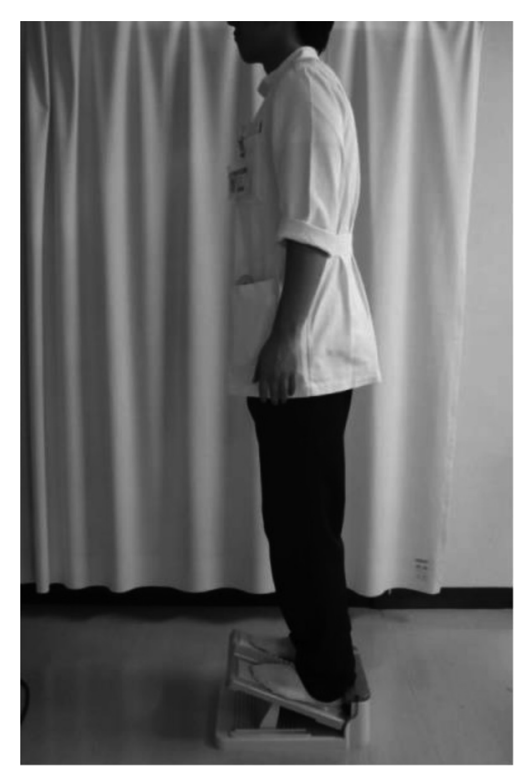

Fig. 1. The slant board used in this study The slant angle was set at 20 degrees

Table 2. Comparison of performance pre- and post-intervention

\begin{tabular}{llcc}
\hline & & Pre-intervention & Post-intervention \\
\hline Comfortable waking velocity & $(\mathrm{m} / \mathrm{s})$ & $0.72 \pm 0.14$ & $0.83 \pm 0.26^{*}$ \\
& $(\mathrm{steps})$ & $22.3 \pm 3.3$ & $20.4 \pm 3.6^{*}$ \\
Maximum waking velocity & $(\mathrm{m} / \mathrm{s})$ & $0.98 \pm 0.32$ & $1.25 \pm 0.31^{*}$ \\
& $(\mathrm{steps})$ & $19.5 \pm 4.2$ & $17.1 \pm 3.3^{*}$ \\
TUG (turn of paralysis side) & $(\mathrm{s})$ & $14.0 \pm 5.3$ & $10.2 \pm 2.3^{*}$ \\
TUG (turn of non-paralysis side) & $(\mathrm{s})$ & $14.0 \pm 5.6$ & $10.1 \pm 2.2^{*}$ \\
\hline
\end{tabular}

steps: number of steps during the 10 -m walking $* \mathrm{p}<0.05$ 
in walking velocity, with a decrease in the number of steps to complete the pre-selected distance. Previous studies indicated that using the slant board the stretches the spastic calf muscles such as gastrocnemius and soleus muscles ${ }^{11)}$. However, this mechanism does not seem to be the only factor responsible for the increased walking velocity observed in our study. Using electromyographic recording, we previously demonstrated that maintenance of standing position with dorsiflexion of the ankle joint in healthy subjects is associated with increased muscle activity of the tibialis anterior (TA) muscle and other anteriorly located leg muscles. The increase in TA muscle activity can produce an anterior shift in the center of gravity. This shift is considered beneficial since it promotes body motion in the anterior direction. Furthermore, the increase in TA muscle activity is expected to strengthen hip joint muscles, such as the rectus femoris muscle, gluteus maximus muscle' and rectus abdominis muscle ${ }^{11)}$. Considered together, it is likely that these mechanisms contributed to the observed improvement in gait function in this study. So far, the "ankle strategy" and "hip strategy" suggested by Horak et al. have been accepted widely as important concepts for rehabilitative management of patients with gait disturbances ${ }^{14)}$. Horak et al. suggest that the hip strategy is used to restore equilibrium when the support surface is smaller than that of the feet ${ }^{14,15}$. Therefore, therapeutic approaches that strengthen the TA muscle seem to be meaningful in clinical settings for stroke rehabilitation, in addition to the above two strategies. This study also confirmed that the use of the slant board improved TUG performance time. The TUG evaluates balance control ability as well as mobility of the lower limbs ${ }^{13}$ ). The observed improvement in TUG performance time suggests that this rehabilitation program improves balance control. Although the mechanism underlying this beneficial effect on balance control remains unknown, strengthening of hip joint muscles is speculated to be responsible. Furthermore, maintaining standing position on the slant board can be considered static activity, in contrast with the dynamic training in stand-up exercise and treadmill exercise. Compared to dynamic training, static training has certain specific characteristics. First, during static training, the therapist can easily assist the patients so that they maintain the standing position. Second, static training using the slant board seems safe. The risk of falling during such training is markedly low, which can encourage the patient to practice daily. These characteristics of static training could have also been involved in the gait function improvement observed in the present study. Further studies using kinematic and/or dynamic gait analyses are warranted to further investigate various aspects of the protocol. We confirmed that the board is safe to use for both patients and health professionals. Therefore, we recommend the application of this program as a home-based rehabilitation program for poststroke hemiparetic patients with gait disturbances of different age groups. The present study has certain limitations. First, the study involved only a small number of patients and lacked a control group. The finding of this study should be confirmed in another randomized controlled study with a larger sample size. Second, the long-term effects of the 30-day training program on walking function remain to be investigated. Third, the duration of board use in one session of the rehabilitation program (3 minute) was arbitrarily selected in this study. In addition, whether the improvement in walking speed and TUG was unrelated to the measure of the other evaluations needs to be clarified. The optimal duration of the rehabilitation program should be investigated, although the duration in the current protocol seems to have produced satisfactory improvement.

The 30-day home-based daily training using the slant board is safe and improved walking function in post-stroke patients with gait disturbances. Further evaluation of the protocol in a large sample is required.

\section{ACKNOWLEDGEMENT}

This study was supported by Tsukuba University Graduate School in 2013.

\section{REFERENCES}

1) Keenan MA, Perry J, Jordan C: Factors affecting balance and ambulation following stroke. Clin Orthop Relat Res, 1984, (182): 165-171. [Medline]

2) Mayo NE, Korner-Bitensky NA, Becker R: Recovery time of independent function post-stroke. Am J Phys Med Rehabil, 1991, 70: 5-12. [Medline] [CrossRef]

3) Kim P, Warren S, Madill H, et al.: Quality of life of stroke survivors. Qual Life Res, 1999, 8: 293-301. [Medline] [CrossRef]

4) Jaracz K, Kozubski W: Quality of life in stroke patients. Acta Neurol Scand, 2003, 107: 324-329. [Medline] [CrossRef]

5) Béthoux F, Calmels P, Gautheron V, et al.: Quality of life of the spouses of stroke patients: a preliminary study. Int J Rehabil Res, 1996, 19: 291-299. [Medline] [CrossRef]

6) Bohannon RW, Andrews AW, Smith MB: Rehabilitation goals of patients with hemiplegia. Int J Rehabil Res, 1988, 11: 181-184. [CrossRef]

7) Harris JE, Eng JJ: Goal priorities identified through client-centred measurement in individuals with chronic stroke: implications for rehabilitation professionals. Physiother Can, 2004, 56: 171-176. [Medline] [CrossRef]

8) Kluzik J, Horak FB, Peterka RJ: Differences in preferred reference frames for postural orientation shown by after-effects of stance on an inclined surface. Exp Brain Res, 2005, 162: 474-489. [Medline] [CrossRef]

9) Nakayama Y, Abo M, Iijima S: Influence on postural sway immediately after standing on a sloping platform—comparison of hemiplegic patients with healthy adults. J Phys Ther Sci, 2008, 23: 585-588.

10) Nakayama Y, Abo M: Influence on maximum anteroposterior displacement of center of pressure immediately after standing on a sloping platform. J Phys Ther Sci, 2009, 24: 387-390.

11) Nakayama Y: Influence of postural strategy on standing on a slope-electromyographic analysis. J Phys Ther Sci, 2009, 24: 817-820. 
12) Buchner DM, Larson EB, Wagner EH, et al.: Evidence for a non-linear relationship between leg strength and gait speed. Age Ageing, 1996 , 25 : 386-391. [Medline] [CrossRef]

13) Podsiadlo D, Richardson S: The timed "Up \& Go": a test of basic functional mobility for frail elderly persons. J Am Geriatr Soc, 1991, 39: 142-148. [Medline] [CrossRef]

14) Horak FB, Nashner LM: Central programming of postural movements: adaptation to altered support-surface configurations. J Neurophysiol, 1986, 55: 13691381. [Medline]

15) Horak FB, Nutt JG, Nashner LM: Postural inflexibility in parkinsonian subjects. J Neurol Sci, 1992, 111: 46-58. [Medline] [CrossRef] 